\title{
Prácticas de Liderazgo en Escuelas con Alta y Baja Cultura Escolar Inclusiva
}

\author{
Leadership Practices in Schools with High and Low Inclusive \\ School Culture
}

René Valdés *

Pontificia Universidad Católica de Valparaíso, Chile

\begin{abstract}
El objetivo de este estudio es describir y analizar la relación entre las prácticas de liderazgo de los equipos directivos y el desarrollo de culturas escolares inclusivas. Para ello se realizó una etnografía escolar durante 30 semanas en dos escuelas con diferentes niveles de desarrollo de culturas inclusivas. Los hallazgos más relevantes indican que las prácticas de liderazgo varían entre ambos centros educativos. En la escuela con alta cultura inclusiva se desarrollaban capacidades profesionales, valores inclusivos, se lideraban procesos de enseñanza y aprendizaje y existía una visión compartida de la inclusión. En el caso de la escuela con baja cultura inclusiva, las prácticas estaban principalmente puestas en la gestión extraescolar de la convivencia escolar. Se discuten los resultados en función de aquellas prácticas promotoras de cultura inclusiva.
\end{abstract}

Descriptores: Liderazgo; Cultura; Integración escolar; Estudio de casos; Inclusión.

The objective of this study is to describe and analyze the relationship between leadership practices of management teams and the development of inclusive school cultures. For this, a school ethnography was carried out for 30 weeks in two schools with different levels of development of inclusive cultures. The most relevant findings indicate that leadership practices vary between both schools. In the school with a high inclusive culture, professional capacities, inclusive values were developed, teaching and learning processes were led, and there was a shared vision of inclusion. In the case of the school with a low inclusive culture, the practices were mainly put into extracurricular management of school coexistence. The results are discussed based on those practices that promote inclusive culture.

Keywords: Leadership; Culture; School integration; Case study; Inclusion.

\section{Liderazgo escolar y desarrollo de culturas escolares inclusivas}

La revisión de las investigaciones sobre liderazgo escolar muestra que las preocupaciones han estado, principalmente, en la importancia de la dirección escolar en los resultados académicos de los y las estudiantes (Weinstein y Muñoz, 2012). Sin embargo, menos profusa es la investigación sobre el papel del liderazgo escolar en el ámbito de la inclusión educativa (Ryan, 2017), y menos aún en las implicancias de los directivos en el desarrollo de culturas escolares inclusivas (Valdés, 2018; Valdés y Gómez-Hurtado, 2019). Una cultura escolar inclusiva, según Booth y Ainscow (2015) se relaciona con la creación de una comunidad escolar segura, acogedora, colaboradora y estimulante, en la que cada persona es valorada, lo cual es la base fundamental primordial para que todo el alumnado

*Contacto: rene.valdes@pucv.cl

ISSN: 0718-7378

www.rinace.net/rlei/
Recibido: 10/04/2020

$1^{\text {a }}$ Evaluación: 03/08/2020

Aceptado: $\quad$ 14/08/2020 
tenga mayores niveles de logro. Se refiere, además, al desarrollo de valores inclusivos, compartidos por todo el personal de la escuela.

Los estudios sobre culturas inclusivas dan cuenta de que su desarrollo permite impulsar el tránsito hacia la educación inclusiva. Autores como Moliner, Sales y Escobedo (2016), Ashikali y Groenevald (2015), Booth y Ainscow (2015) y Dorczak (2013) concuerdan en que se puede reconocer una escuela inclusiva por la cultura que esta tenga. En ese sentido, no se puede pensar la transformación de una institución sin la modificabilidad del ámbito cultural. Según la guía para el desarrollo de escuelas inclusivas (Index for Inclusion) en todas sus versiones, la cultura inclusiva es una de las tres dimensiones para el cambio escolar, junto a prácticas y políticas inclusivas, aunque estas dos últimas están altamente influenciadas por el cambio cultural que viva una escuela.

Ahora bien, ¿quién desarrolla una cultura inclusiva? y ¿cómo se desarrolla? La literatura especializada parece tener más respuestas a la primera que a la segunda pregunta, señalando que las prácticas directivas son claves para instalar y promover una cultura de la inclusión (Ashikali y Groeneveld, 2015; León, Crisol y Moreno, 2018; Ryan, 2017; Valdés, 2018; Valdés y Gómez-Hurtado, 2019; Waldron y Mcleskey, 2010). Los estudios de Gómez-Hurtado (2013, 2014) dan cuenta de que, más que los directores de escuela son los equipos directivos los encargados de desplegar prácticas inclusivas que promuevan también una cultura de la inclusión. En la misma línea, de León (2012) y de León et al. (2018) mencionan que el director de una escuela es clave, pero insuficiente por sí mismo: pues conviene que se apoye en equipos comprometidos con la inclusión para producir cambios significativos en las culturas escolares. Según Beneke y Cheatham (2016), la escuela debe hacer suyo un punto de vista inclusivo y abordarlo desde la cultura, pues permite estar mejor preparada para responder a la diversidad de la escuela. Sin embargo, el cambio cultural de una institución debe estar claramente intencionado por parte de los líderes, pues es la forma más segura de mantener la cultura (Celik, Ashikali y Groeneveld, 2013). Punto coincidente con Boekhorst (2015), quien explicita que una institución comprende varios procesos de esfuerzo para alcanzar la inclusión, siendo uno de los más relevantes, la aparición de auténticos líderes que modelen la transformación deseada de una institución.

En este escenario, resulta relevante no solo dar cuenta de si las culturas escolares inclusivas están supeditadas al liderazgo que tenga un centro escolar, sino dar cuenta de qué prácticas despliegan los equipos directivos para promoverla y desarrollarla.

\section{El liderazgo escolar como práctica}

En los últimos años, los estudios sobre liderazgo escolar han estado enfocados principalmente en las prácticas en lugar de las competencias (Ministerio de Educación, 2015). Algunos autores (Carroll, Levy y Richmond, 2008; Spillane y Ortiz, 2017; Uribe y Celis, 2012) mencionan diferencias importantes entre práctica y competencia en el ámbito del liderazgo. Principalmente sostienen que las prácticas son compartidas, contextualizadas y flexibles. A diferencia de las competencias, que son individuales y que suponen que funcionan en todo contexto sin considerar la acción situada. Para el caso de este estudio, se asume el concepto de práctica desde los planteamientos de Wenger (2001). 
Según Wenger (2001), participar de una comunidad de práctica significa poner a disposición los recursos personales para el logro de objetivos compartidos. Esto se traduce en que aprender es participar de una comunidad y esto implica construir una identidad que también es compartida.

Según Leithwood, Harris y Hopkins (2008), el liderazgo escolar es el segundo factor que más influye en el aprendizaje de los estudiantes (después del trabajo en aula) y que no depende precisamente del carisma, sino de las prácticas, entendiendo el liderazgo como la labor de movilizar e influenciar a otros para articular y lograr los objetivos y metas compartidas (Leithwood et al., 2008). De esta forma, el liderazgo escolar se configura como un conjunto de prácticas que presenta variaciones de acuerdo al contexto, vulnerabilidad y tipo de establecimiento (Uribe y Celis, 2012).

El concepto de prácticas de liderazgo comprende un constructo dinámico y contextualizado (Uribe y Celis, 2012), acorde a las comunidades de práctica. Según Spillane y Ortiz (2017) las prácticas de liderazgo surgen a partir de las interacciones entre los miembros de una escuela en función de los intereses comunes y considerando el contexto. Es decir, los individuos actúan, pero lo hacen en relación a otros. Así, la práctica se constituye a partir de una colaboración entre las personas que trabajan en una organización, la cual está influida por el entorno. Desde esta perspectiva, el liderazgo es la práctica del mejoramiento (Elmore, 2010) y no se trata de atributos personales, sino de un conjunto de acciones que pueden ser enseñados y aprendidos. En ese sentido, en este estudio se complementan las perspectivas de Spillane y de Elmore, entendiendo el liderazgo como la práctica del mejoramiento inclusivo, dando hincapié en el desarrollo de culturas inclusivas y en la participación de todos los actores escolares, sin dejar de lado el rendimiento académico, sino más bien complementándolo.

En síntesis, esta investigación presenta tres focos que conviene precisar: (1) no está basado en las competencias, sino en las prácticas, (2) focaliza el interés en los equipos directivos y no solo en los directores y (3) no está basado en la gestión, sino en el liderazgo escolar. De esa manera, el objetivo es describir y analizar la relación entre las prácticas de liderazgo de los equipos directivos y el desarrollo de culturas escolares inclusivas.

\section{Metodología}

De acuerdo al objetivo de este estudio, se opta por un diseño cualitativo de investigación y por el método de etnografía escolar. Según Díaz de la Rada (2013) la etnografía escolar debe, de forma irrenunciable, develar las prácticas de una escuela y desentrañar las manifestaciones culturales que allí habitan. El proceso de etnografía escolar duró siete meses y se desarrolló en dos escuelas consideradas casos extremos.

\section{Casos de estudio y selección de participantes}

Para seleccionar los dos casos de estudio se aplicó una encuesta validada de prácticas de convivencia inclusiva (López y Valdés, 2018; Valdés, López y Chaparro, 2018) a 65 escuelas de la región de Valparaíso de Chile. Si bien el instrumento evaluó múltiples dimensiones, se consideró aquella que medía cultura inclusiva para adultos (directivos, profesores, profesionales no docentes y familias) y aquella que medía convivencia inclusiva para estudiantes. Se seleccionaron las escuelas según la posición de estas en la frecuencia de prácticas reportadas $(1=$ la escuela con más frecuencia y $65=$ la escuela con menos frecuencia). La escuela 1 presentó alta convivencia escolar inclusiva según estudiantes (2 
de 65) y alta cultura inclusiva según las familias (10 de 65) y según profesionales (16 de 65). La escuela 2 presentó baja convivencia inclusiva según estudiantes (32 de 65) y baja cultura inclusiva según las familias (43 de 65) y según profesionales (35 de 65). Ambas escuelas seleccionadas pertenecen a la zona norte de la región de Valparaíso. Antes de seleccionar los dos casos, se constató que ambas escuelas fuesen gratuitas, sin selección escolar y que tuviesen a la inclusión como uno de los objetivos de su proyecto educativo institucional (PEI), que es un documento público y oficial. Estas características fueron los criterios utilizados para seleccionar a los centros escolares como casos de estudio. Por último, es necesario señalar que ambas escuelas presentan alta vulnerabilidad escolar y se encuentran en barrios de alto riesgo social.

\section{Trabajo de campo}

Para la producción de datos se realizaron observaciones participantes, entrevistas individuales, grupos focales y revisión de documentos. La inmersión etnográfica duró siete meses en ambas escuelas. Se realizaron entre 30 y 32 visitas durante 30 semanas a cada una de las instituciones. El proceso fue secuencial y progresivo: los primeros tres meses fueron de observación participante en los espacios formales (reuniones, sala de clases, consejos, etc.) e informales de la escuela (patios, espacios de recreación, comedores). Toda vez que se estaba en las escuelas se tomaban notas en el diario de campo. Luego, durante dos meses, se realizaron entrevistas individuales a todos los participantes de la escuela, aunque principalmente al equipo directivo. En estas entrevistas se pusieron como temas de conversación los elementos encontrados en la parte de observación. Finalmente se hicieron grupos focales, en los cuales se trabajó con los resultados de las entrevistas. De esa forma, la etnografía fue secuencial, recursiva y a la vez los mismos participantes eran árbitros de calidad en la producción de información (Flick, 2012). Paralelamente se revisaron los documentos relevantes para las escuelas (proyecto educativo institucional, manual de convivencia, diversos protocolos de acción y reglamento escolar).

\section{Procedimiento de análisis, validez y confiabilidad}

Para analizar los datos producidos en la fase etnográfica se utilizó el análisis de contenido categorial-temático (Vásquez, 1994). Este análisis busca descomponer los textos en unidades para luego proceder al agolpamiento de categorías de trabajo. Este trabajo se resume de la siguiente manera: (1) selección y lectura de documentos primarios, (2) codificación prestablecida de citas, (3) codificación emergente de citas, (4) categorización según objetivo del estudio, (5) depuración de las bases hermenéuticas, agrupando códigos y eliminando otros, (5) relacionar las categorías con las notas de campo, (6) relacionar las categorías con la revisión documental, (7) selección de categorías finales según densidad de citas y relación entre técnicas, (8) triangulación metodológica y (9) redacción de resultados preliminares. Según Flick (2012), la investigación cualitativa debe dar garantías de validez y plausibilidad. Para el alcanzar este propósito se llevó a cabo una triangulación metodológica. Esta implica alcanzar resultados similares utilizando diversas técnicas y en diferentes momentos (tiempo y espacio). Esto quiere decir que las categorías que se exponen en los resultados aparecieron de forma transversal en todas las técnicas aplicadas.

\section{Consideraciones éticas}

Finalmente, para la inmersión etnográfica y para el desarrollo de las diversas técnicas, se solicitaron autorizaciones a los directores de escuelas, consentimientos informados a los 
adultos y asentimientos informados a los estudiantes. Todos los participaron decidieron ser parte de la investigación.

\section{Resultados}

Los resultados se exponen de la siguiente manera; primero, para ser fiel a la etnografía escolar, se describe en primera persona la experiencia etnográfica del autor en las escuelas y se describen elementos claves de cada una de las instituciones. Luego, en función de las categorías seleccionadas del análisis de contenido, se describen las prácticas de liderazgo (cuadro 1).

Cuadro 1. Identificación y definición de las categorías de resultados

\begin{tabular}{|c|c|c|c|}
\hline \multicolumn{2}{|c|}{ ESCUELA 1} & \multicolumn{2}{|r|}{ ESCUELA 2} \\
\hline Categorías & Definición & Categorías & Definición \\
\hline $\begin{array}{l}\text { Desarrollo de } \\
\text { capacidades } \\
\text { profesionales }\end{array}$ & $\begin{array}{l}\text { Se refiere a la detección } \\
\text { de talentos, de la } \\
\text { contratación y } \\
\text { permanencia de } \\
\text { profesionales } \\
\text { calificados según los } \\
\text { criterios de la dirección } \\
\text { (Mineduc, 2015). }\end{array}$ & $\begin{array}{l}\text { Gestión de la } \\
\text { convivencia }\end{array}$ & $\begin{array}{l}\text { Guarda relación con todas las } \\
\text { acciones que han desplegado } \\
\text { los directivos para tener un } \\
\text { buen clima escolar, aunque a } \\
\text { través de estrategias de tipo } \\
\text { extraescolar. } \\
\text { Es decir, relacionadas con la } \\
\text { celebración de festividades y } \\
\text { conmemoraciones. }\end{array}$ \\
\hline $\begin{array}{l}\text { Valores } \\
\text { inclusivos }\end{array}$ & $\begin{array}{l}\text { Se trata de un } \\
\text { compromiso de los } \\
\text { directivos con valores } \\
\text { particulares que } \\
\text { representan el deseo de } \\
\text { superar la exclusión y } \\
\text { promover la } \\
\text { participación (Booth y } \\
\text { Ainscow, 2015). }\end{array}$ & $\begin{array}{l}\text { Gestión de la } \\
\text { participación } \\
\text { escolar }\end{array}$ & $\begin{array}{l}\text { Los equipos directivos } \\
\text { implementan prácticas que } \\
\text { facilitan espacios para la } \\
\text { participación de la } \\
\text { comunidad escolar, } \\
\text { (Mineduc, 2015). } \\
\text { En el caso de esta escuela, la } \\
\text { participación es el sello que } \\
\text { el director desea darle a la } \\
\text { institución. }\end{array}$ \\
\hline $\begin{array}{l}\text { Liderazgo de } \\
\text { los procesos } \\
\text { de } \\
\text { enseñanza }\end{array}$ & $\begin{array}{l}\text { Los equipos directivos } \\
\text { guían, dirigen y } \\
\text { gestionan eficazmente } \\
\text { los procesos de } \\
\text { enseñanza y } \\
\text { aprendizaje (Mineduc, } \\
\text { 2015). }\end{array}$ & $\begin{array}{l}\text { Gestión de las } \\
\text { redes de } \\
\text { apoyo }\end{array}$ & $\begin{array}{l}\text { Se trata del trabajo } \\
\text { cooperativo con otras } \\
\text { organizaciones que } \\
\text { interactúan con la escuela y } \\
\text { que pueden servir de apoyo } \\
\text { al centro escolar y } \\
\text { viceversa. }\end{array}$ \\
\hline $\begin{array}{l}\text { Visión } \\
\text { compartida } \\
\text { de la } \\
\text { inclusión }\end{array}$ & $\begin{array}{l}\text { Los directivos lideran la } \\
\text { construcción o } \\
\text { actualización de una } \\
\text { visión estratégica e } \\
\text { inclusiva sobre el } \\
\text { establecimiento y sus } \\
\text { objetivos, promoviendo } \\
\text { que esta sea } \\
\text { comprendida y } \\
\text { compartida (Mineduc, } \\
\text { 2015). }\end{array}$ & & \\
\hline
\end{tabular}

Fuente: Elaboración propia. 


\subsection{Escuela 1}

La escuela 1 es gratuita y no cuenta con procesos de selección escolar. Posee un equipo directivo estable: los mismos integrantes llevan cinco años juntos. Todos los estamentos de la escuela tienen un discurso a favor de la inclusión y con alta motivación por la transformación escolar. Tienen una alta presencia en la escuela y su líder clave no es la directora, sino la jefa de unidad técnico pedagógica. Esto quiere decir que ella representa y lidera el principal objetivo de la escuela: desarrollar aprendizajes en todos los estudiantes. Esta institución actualmente se encuentra en una fase pedagógica, es decir, que en general sus preocupaciones y prioridades están en desarrollar aprendizajes significativos en sus estudiantes.

Mi primer día en la escuela 1 resultó ser la antesala de una agradable experiencia etnográfica. La directora me recibe con una broma. Nos reímos juntos. Entramos a su oficina, nos tomamos un café, hablamos de trivialidades y luego le explico mi presencia en la escuela. Asume orgullosa mi rol de etnógrafo y me da la bienvenida a la institución. El resto de la experiencia en la escuela es igual de positiva. Fueron siete meses intensos de vistas semanales, de acceder a la sala de clases, a reuniones, conversar con estudiantes, profesores, familias y directivos. Incluso no necesitaba planificar las visitas: yo era o parecía uno más de la escuela. Un día viernes el profesor de matemáticas bromea y me dice "ya eres parte de la casa. Uno más entre nosotros" (nota de campo 1). Durante mi estadía en la escuela escuché problemas, sugerí soluciones, me pidieron opiniones, recibí invitaciones a almorzar, asistí a festividades e incluso fui jurado en un concurso de tortas.

Una de las frases que escuchaba con frecuencia en la escuela era "somos como una familia" (nota de campo 2). Esta frase, tan característica de culturas escolares acogedoras, es clave para entender mi paso por la institución; la confianza y la buena comunicación entre profesores, directivos y profesionales no docentes era la evidencia de que mi próspero paso por la escuela no fue una cuestión fortuita, sino que respondía a una forma de vida institucional.

\subsubsection{Desarrollo de capacidades profesionales}

Una de las primeras frases que emitió la directora de la escuela 1 y que luego se reproduciría entre otros actores era "en esta escuela contratamos buenas personas" (Nota de campo 3). Al comienzo no entendí bien, pues la idea de buenas personas me resultó, en cierto punto, abstracta. Luego lo comprendí en los grupos focales cuando los profesores me dijeron que "en este tipo de escuela se necesita cierto perfil de persona, no cualquiera puede trabajar en este contexto, de pobreza, marginalidad y vulnerabilidad" (nota de campo 4). La directora sabía esto de antemano, lleva 30 años en la escuela, y ha visto cómo muchos profesionales han pasado de forma fugaz por la escuela. Cuando la directora contrata profesionales intenta resguardar ciertos atributos personales y profesores que, según ella, podrían favorecer su trabajo en la institución.

$$
\begin{aligned}
& \text { To creo que tiene que trabajar la gente con el perfil que el establecimiento propone, } \\
& \text { porque hay un perfil de profesores (...) el perfil más importante es del profesor, que } \\
& \text { sea proactivo, que sea atento, que logre relaciones de amor, no sé si llamarlo de amor, } \\
& \text { que los niños se sientan que son queridos por el profesor, que no haya conflicto, que } \\
& \text { sean acogedores con los apoderados. (Directora, escuela 1) }
\end{aligned}
$$

Este abordaje se complementa con la reorganización de profesionales en distintos puestos y la detección de talentos que realiza la jefa de UTP. Esta profesional cuando intuye que una persona puede desempeñarse mejor en otro puesto, realiza cambios, siempre por 
medio del consenso. En la misma línea, como dupla directiva, han sido capaces de detectar ciertos talentos y aprovecharlos. Por ejemplo, han movilizado profesores talentosos a cursos difíciles con la intención de enfocar los esfuerzos en el aprendizaje escolar y fortalecer el vínculo docente-alumno. La actual inspectora (o supervisora interna a cargo principalmente del alumnado y sus familias) es una profesional sin estudios en pedagogía, pero, en palabras de la directora "una persona con habilidades emocionales increíbles, ella apaga todos los incendios” (nota de campo 5). En ese sentido, tanto el foco en contratación de personas, como reorganización de puestos y detección de talentos, han potenciado el desarrollo de capacidades profesionales del personal de la escuela. Es necesario mencionar que en los últimos dos años el equipo directivo ha gestionado 12 capacitaciones a sus docentes, inspectores y directivos en temas como evaluación curricular y Diseño universal de aprendizajes para docentes y en temas de conductas disruptivas y gestión de la calidad educativa para inspectores y directivos.

\subsubsection{Valores inclusivos}

Mi cómoda experiencia etnográfica en la escuela se debe, en cierto punto, al ambiente familiar y valórico de la escuela. Esto se manifestaba no solo en los valores que intencionadamente promovían los directivos, sino en las preocupaciones de la escuela para atender a la diversidad. Es decir, el equipo directivo defendía algunas prácticas escolares por los valores que estaban implicados en ellas. Valores como los derechos, el amor, la honestidad y la confianza eran particularmente impulsados por la directora de la escuela y aceptados y amparados por el resto del personal.

\section{Sí, la calidad humana de la gente, el trabajo colaborativo, el complemento que se hace de todo (...) porque todos nos tenemos que hacer cargo de todo ¿̨cachai'? ', todos tenemos la confianza de dirigirnos a un superior, por ejemplo (...) Un trabajo de verdad de mucha confianza, muy en equipo, y si algo no resulta, súper autocrítico, si el trabajo era de todos, jamás echando la culpa. $\Upsilon$ lo toman siempre como una manera de mejorar. (Jefa de UTP escuela 1)}

Además, la directora tomaba decisiones considerando valores. Si no estaba segura de una decisión, se guiaba inspirada por los valores antes de tomarla o por los valores del proyecto educativo de la escuela. Esto implicaba entonces que las actividades de la escuela contenían en sí mismas un conjunto de valores. En esa misma línea, a la jefa de UTP le interesaba que las decisiones curriculares contemplaran ámbitos valóricos y actitudinales, decía que "potenciar los valores también es trabajar el área pedagógica" (nota de campo 6). Por último, la misma inspectora/supervisora de la escuela, que es la encargada de mantener la disciplina dentro del centro, mencionó en una entrevista que "uno de los obstáculos para la inclusión escolar era que a veces se olvidaban los valores" (nota de campo 7).

\subsubsection{Liderazgo de procesos de enseñanza y aprendizaje}

Esta escuela se encuentra actualmente focalizando su atención en un ámbito pedagógico. Esto quiere decir que todas las energías se encuentran en que todo el alumnado pueda aprender, en desarrollar las habilidades instrumentales en los primeros niveles, en ampliar la oferta metodológica en la sala y en dar respuesta a los estándares curriculares. Si bien en Chile se anhela un liderazgo pedagógico asumido por la dirección, en esta escuela ese rol lo lidera y desarrolla la jefa de la unidad técnica pedagógica. Es ella quien lidera los procesos, quien orienta y quien organiza la escuela en función de las prioridades

\footnotetext{
${ }^{1}$ Modismo chileno que significa “¿comprendes?” o “¿entiendes?”.
} 
pedagógicas. En este caso, la directora apoya el discurso, pero no asume prácticas concretas de abordaje pedagógico. Incluso ella menciona que "ahora tenemos a una persona con respaldo, un perfil técnico" (nota de campo 8) aludiendo al rol que realiza la jefa de UTP. Esta profesional ve su trabajo en la escuela de la siguiente manera:

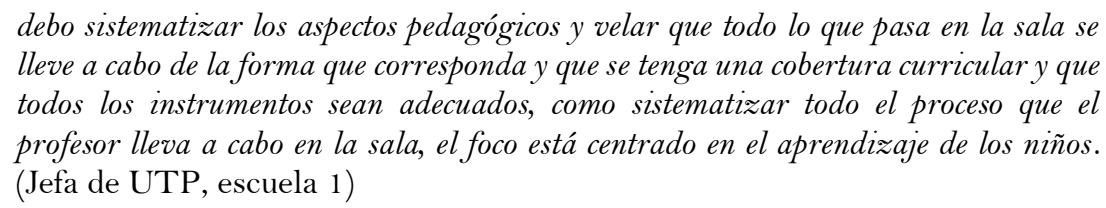

Ahora bien, una de las características claves de esta fase pedagógica que lidera la jefa de UTP es el enfoque pedagógico basado en el contexto y no en el déficit. La escuela pone las prioridades en qué pueden hacer los profesores y directivos con los estudiantes y no descansa en un discurso estático de que son los alumnos el problema. El facilitador se encuentra en poner la atención en las prácticas escolares y no solo en el estudiante o su contexto. Esto visibiliza el rol del profesor (o de la escuela) y ubica las atribuciones en las prácticas de aula y no solamente en la vida de los estudiantes. Por ejemplo, los docentes llevan tiempo trabajando con adecuaciones curriculares, diseño universal de aprendizaje y fortaleciendo el vínculo afectivo con los estudiantes.

\subsubsection{Visión compartida de la inclusión}

La presencia de un "lenguaje común" es uno de los grandes facilitadores si pensamos en una institución con orientación inclusiva. Con la presencia de un lenguaje común se entiende que toda la escuela comparte un discurso que refleja un objetivo y una meta compartidos por todos los estamentos de la institución. Este discurso se materializa en una visión de escuela impulsado por la directora mediante su forma de ver la institución y llevado a la práctica por la jefa de UTP en el abordaje con los profesionales de la escuela. Cada estamento de la escuela cumple funciones y roles específicos que, desde sus deseos para la escuela, se relacionan entre sí. Las preocupaciones de los profesores son las mismas preocupaciones que asistentes de la educación o directivos. Todos buscan la mejora en los aprendizajes y sostener relaciones humanas amenas y afectuosas y aunque los profesionales desde sus cargos tengan roles distintos, existe un encuentro entre todos ellos. Esta visión ha impregnado en los profesionales hasta el punto de no querer movilizarse de la escuela:

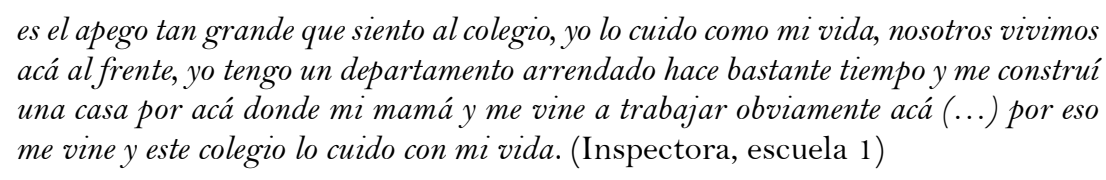

Esto refuerza las posibilidades de crear una comunidad de práctica (Wenger, 2001), donde la identidad y el aprendizaje sean consecuencias de la toma de decisiones y de las motivaciones que movilizan a sus actores. En este contexto el rol de la directora es relevante pues, como sostenedora, es quien subraya el ideal que la escuela persigue. No obstante, al igual que en las prácticas antes señaladas, la jefa de UTP es quien lleva esta visión al aula mediante el liderazgo pedagógico, revisando el proyecto educativo de la escuela, comunicando los objetivos de la escuela y estimulando una cultura de la inclusión.

\subsection{Escuela 2}

La escuela 2 es una escuela con un equipo directivo inestable: en los últimos cinco años han tenido 3 directores y 2 jefes de UTP. El director tiene un foco en la mejora de las 
relaciones humanas entre los adultos, mientras que el jefe de UTP aboga por la presencia de docentes afectivos en el aula. Esto implica que se visualiza un foco parcialmente compartido en la importancia del área socioemocional y convivencial entre los miembros del equipo. Esta institución se encuentra en una fase convivencial, es decir, los intereses y las acciones están puestos en mejorar las relaciones humanas y crear una comunidad escolar participativa.

Mi primer día en la escuela 2 también marca lo que sería el resto de mi estadía en la institución: el director me recibe distante, desconfiado, pero a medida que avanza la conversación va mejorando su disposición. Esto no implica una relación estrecha, pero al menos me permite estar en la escuela sin sentirme incomunicado. En ese sentido, la escuela 2 es distinta a la escuela 1 y esto se refleja, principalmente, en no hacer sentir al otro bienvenido:

Los adultos no hacen eso, el buen trato es como que falta, yo no siento que acá llegue un practicante o una persona que viene a reemplazar y que se sienta cómodo, como que es responsabilidad del que llega integrarse, la comunidad no hace nada por integrarlo. (Encargada de convivencia, escuela 2)

La cita anterior ilustra mi propio sentir en la escuela. No sentirse bienvenido implicaba estar en terrenos ajenos: sola una vez ingresé a la sala de profesores y fui acompañado por una docente. Si bien ingresé a varios cursos a observar clases, los profesores no se interesaban por mi presencia. Me sentía satisfecho si podía sostener con ellos una conversación de 10 minutos. A diferencia de la escuela 1, las confianzas estaban fragosas y la buena comunicación no era una cualidad de esta organización. Una vez ingresé a un consejo de profesores y nadie me presentó. A veces -en invierno- esperé en el patio reuniones que estaban agendadas y que nunca se concretaron: nadie me preguntaba si me habían atendido, nadie me ofrecía un café o una sala donde esperar. En resumen, no fue fácil sentirse cómodo en la escuela 2. Yo me volví guía de mis propios procesos de inserción en los diversos espacios de la institución y, sobre todo en ese contexto, fui lo más parecido a un turista impertinente.

\subsubsection{Gestión de la convivencia escolar}

La celebración de hitos, festividades y conmemoraciones es un elemento bien valorado por la escuela en general. El esfuerzo por llevar a cabo diversas celebraciones y festividades, como fiestas patrias, el día de la familia, día del alumno, día del profesor, bienvenida a clases, etc., es bien estimado por los diferentes estamentos de la escuela. Esto ha sido señalado, especialmente, por el director de la escuela como una práctica que ha permitido integrar a todos los actores de la escuela. El mismo director señala que uno de sus grandes intereses es potenciar las relaciones humanas, la lógica de escuela comunitaria, la reciprocidad con vecinos y el bienestar de todas las personas al interior de la institución. Incluso entre sus primeras medidas fue el de crear un comité de convivencia escolar:

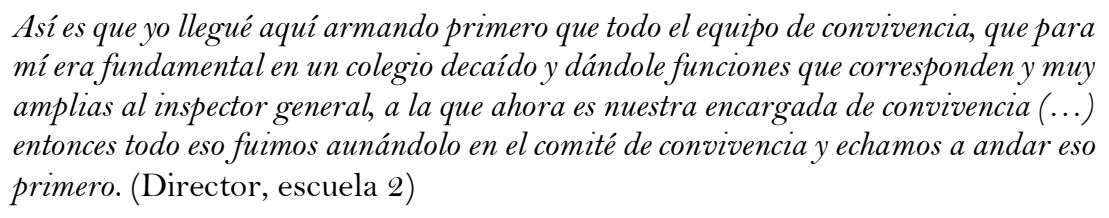

En ese sentido, el director focaliza sus propósitos en generar instancias de cooperación y comunidad escolar. Los estudiantes y familias también identifican estos eventos como 
positivos para la escuela, pues advierten que le hace bien a la escuela. Si bien una escuela con orientación inclusiva debe ser capaz de tener tradiciones que potencian la identidad de la institución, en el caso de esta escuela, las celebraciones son vistas como instancias para mejorar las relaciones interpersonales. Este abordaje se materializa en un actor importante: la encargada de convivencia escolar. Así como en la escuela 1, la jefa de UTP es relevante en la fase pedagógica de la institución, en este caso, es la encargada de convivencia quien se vuelve un actor clave para los propósitos de la institución educativa. Su rol en el equipo de gestión le ha permitido tener una visión panorámica sobre las prácticas de la escuela, ganándose la confianza del equipo directivo. Esta profesional ha sido además potenciada por el director de la escuela. De esta manera, gran parte de las energías que utiliza la escuela van dirigidas a mejorar las relaciones humanas, principalmente entre adultos. En ese sentido, las acciones del equipo directivo en conexión con la encargada de convivencia han permitido tomar medidas, consensuar, liderar y gestionar decisiones que sean vinculantes con la mejora escolar.

\subsubsection{Gestión de la participación escolar}

Como esta escuela se encuentra en una fase de mejora de la convivencia, uno de los retos del director es promover la participación de todos los actores. Fue él quien puso en el proyecto educativo institucional el sello de la participación. El director menciona que "me gusta una escuela en que todos participen y se conecte con la comunidad" (Nota de campo $\mathrm{N}^{\circ}$ 8). Es necesario mencionar que el director tiene una experiencia previa de concejal en el gobierno local del municipio. En ese sentido, su deseo viene antecedido por un trabajo cercano con las personas y con la localidad de la ciudad en la que se encuentra la escuela:

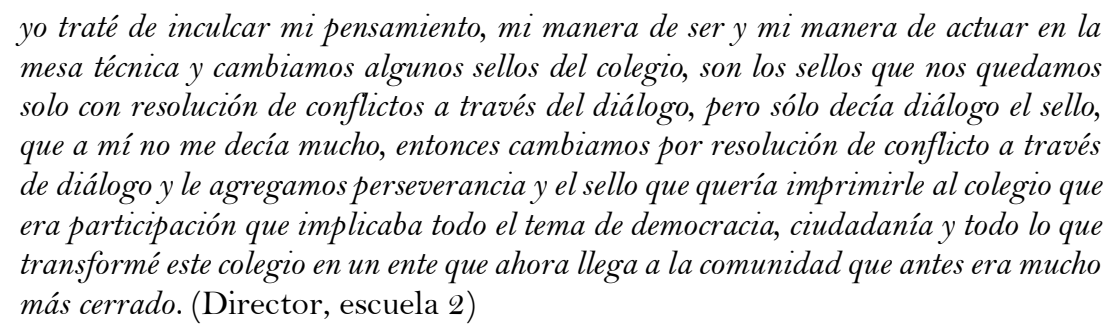

$\mathrm{El}$ director ha logrado gestionar consejos escolares informativos y resolutivos, lugar donde se han podido tomar decisiones en conjunto como en qué usar algunos dineros. Lo mismo con estudiantes, los cuales, mediante apoyo directivo, han postulado a concursos municipales con el propósito de participar de instancias interescolares. Si bien es el director quien principalmente intenta promover la participación de todos y todas, es el jefe de UTP quien asume un rol con la participación familiar. Los profesores me comunicaron que, si bien al director le preocupa la participación, señalan que no siempre sus acciones son las más adecuadas en ese sentido, mencionando que es más un discurso que un conjunto de acciones.

\subsubsection{Gestión de las redes de apoyo}

La conexión con otras instituciones, sea de la comuna o fuera de ella, es un elemento también altamente valorado por la institución. No solamente redes como carabineros y hospitales, sino con iglesias, fundaciones y organismos no gubernamentales que colaboran de cierta manera en el bienestar estudiantil. Esto es especialmente valorado por equipo directivo y por profesores, quienes señalan que el director cuenta con diversos contactos que pueden facilitar la optimización de recursos y aprovechar instancias para que familias 
y estudiantes accedan a servicios que no siempre pueden contar en su vida cotidiana, como acceso a médicos, nutricionistas, etc.:

\begin{abstract}
las redes de apoyo, eso también forma parte de una de mis virtudes, yo tengo mis contactos, tengo la facilidad de buena llegada con las instituciones, con la gente, me llevo bien con todos, no me peleo con nadie (...) yo fui concejal entonces yo tengo una red ahí importantísima (...) yo llamo por teléfono y me vienen a buscar lo que yo quiera, quiero aquí, tengo un acto y me traen un escenario grande, eso no lo hacen otros colegios, es por mis redes, por mis amigos. (Director, escuela 2)
\end{abstract}

Estas redes de apoyo también permiten ubicar a la escuela como centro de acceso para instituciones que desean ocupar las instalaciones o dependencias de la institución: contactos con iglesias, fundaciones, ONG's, etc. Tener redes de apoyo facilita la función directiva en cuanto permite ampliar las funciones de la escuela y responder a las diversas necesidades de estudiantes y familias. Sobre esta categoría de trabajo, queda la sensación que el director utiliza la escuela como plataforma política y de visibilización ante la localidad.

\title{
5. Conclusión y discusiones
}

El problema base de este estudio es que, si bien una cultura inclusiva es clave para una escuela que persigue la inclusión, la información es menos profusa y más dispersa en relación a las prácticas concretas para promoverla y desarrollarla. Con base a esta información, el objetivo de este este estudio fue describir las prácticas de liderazgo de los equipos directivos y, a la vez, analizar la relación de estas con el desarrollo de culturas escolares inclusivas en dos escuelas de la región de Valparaíso. La primera escuela presentaba más desarrollo de una cultura de la inclusión. La segunda escuela, era su contraste, y tenía menos desarrollada la cultura inclusiva. Ambas escuelas sirven como marcos interpretativos para relacionar liderazgo e inclusión educativa.

El equipo directivo de la escuela con alta cultura inclusiva focalizaba sus energías en desarrollar un marco de valores como escenario de los procesos de inclusión. Para esto desarrollaba capacidades profesionales y buscaba instaurar un lenguaje común, una visión de equipo que permita permear el conjunto de prácticas pedagógicas (Valdés y GómezHurtado, 2019). Según los planteamientos de Booth y Ainscow (2015) mientras la inclusión no sea una cuestión de valores, se corre el riesgo de que sea una moda educativa. Este planteamiento ha sido entendido por el equipo directivo quien, a través de las prácticas antes mencionadas, ha podido conducir la institución a alcanzar un ideario inclusivo. Si bien el liderazgo lo asume principalmente la jefa de UTP, hay que mencionar que la directora aporta con un marco de creencias a favor de la inclusión que permite abrir camino a las prácticas de la unidad técnica pedagógica. El liderazgo que asume la jefatura técnica lo podemos encuadrar en lo que Thorpe y Bennett-Powell (2014) llaman liderazgos medios, los cuales implican a aquellas personas que, no siendo director o directora, cumplen un rol troncal en la mejora de las escuelas. En el mismo sentido, los estudios de Ryan (2017) mencionan que los directivos con enfoque inclusivo focalizan sus compromisos en la comunicación y en la colaboración con todo el personal de la escuela, potenciando a aquellos que pueden ser relevantes para la transformación escolar.

En el caso de la escuela con una cultura inclusiva menos desarrollada, posee un equipo directivo inestable y sus miembros cuentan con objetivos distintos. En parte, esto ha permitido que la escuela se encuentre con las relaciones humanas fragmentadas. Por este 
motivo, las prácticas del equipo directivo han estado focalizadas en reconstituir las prácticas de la escuela. Para ello han desplegado acciones en la participación, convivencia y redes de apoyo como focos de acción de la mejora de las relaciones humanas. Los estudios realizados por la Agencia de Calidad de la Educación (2017) aclaran que aquellas escuelas que cuentan con un liderazgo inicial son escuelas que están preocupadas por los temas de convivencia y clima escolar. Desde esa perspectiva, no solo se entiende la convivencia como vía para los aprendizajes, sino además para empezar a focalizar las energías en el ámbito pedagógico.

A pesar de lo dicho anteriormente, pareciera que las escuelas de este estudio se encuentran en fases o trayectorias distintas (Bellei, Valenzuela, Vanni y Contreras, 2014). Según los estudios de la práctica, el contexto, la situación y la época son componentes de la práctica (Wenger, 2001). Es decir, no podemos solo mirar las acciones aisladas sin considerar que existe un fundamento histórico y sociocultural. En el caso de la escuela 1, se encuentra en una etapa pedagógica y de relaciones humanas consolidadas, por lo tanto, sus acciones se orientan desde esa línea, siendo la gran líder la jefa de UTP. En el caso de la escuela 2, sus esfuerzos están puestos en la mejora de las relaciones humanas, pues es el área más débil y, en ese sentido, uno de los liderazgos importantes lo asume la encargada de convivencia escolar.

Sin embargo, si contrastamos las prácticas de liderazgo en ambas escuelas, encontramos que la escuela 1 ha dado con los liderazgos acordes al contexto que la institución necesita. $\mathrm{Y}$, desde esa perspectiva, ha sido la directora la gestora y responsable de armar y consolidar un equipo estable de trabajo. Según los estudios realizados desde la cognición distribuida (Spillane y Ortiz, 2017) y desde los liderazgos inclusivos (Ryan, 2017) las prácticas son un conjunto de acciones coproducidas. No hay un gran héroe que guie y modele la acción. En el caso de la escuela 2, es una institución que no ha logrado consolidar un liderazgo ni un equipo de trabajo acorde a las preocupaciones y a la visión de la institución. Esto es necesario señalarlo para no caer en la tentación de hablar de escuelas buenas y malas, sino hablar de escuelas que se encuentran en fases y etapas distintas, en que tanto los líderes como las preocupaciones van a varían según el contexto y los propósitos institucionales (Bellei et al., 2014).

Si nos focalizamos en prácticas concretas de promoción de cultura inclusiva, hemos identificado tres prácticas específicas: desarrollo de capacidades profesionales, valores inclusivos e instauración de una visión compartida de la inclusión. La primera ha permitido no solo que los docentes se sientan bien, sino que ha aumentado la confianza entre profesionales, un valor relevante en el ámbito de una cultura inclusiva (Booth y Ainscow, 2015). La segunda ha permeado el total de prácticas escolares de la institución y, por lo tanto, se presenta como un elemento central en el mantenimiento de culturas inclusivas, Y la tercera, ha logrado crear una comunidad escolar cohesionada. Esto se visualiza en la presencia de un "lenguaje común" al interior de la institución escolar. Si bien no podemos decir que la escuela 1 tiene un liderazgo inclusivo (y tampoco decir que la escuela 2 tiene un liderazgo excluyente), sí podemos decir que existe un camino, una orientación.

No obstante, hay un elemento diferenciador: la gestión pedagógica de la escuela 1 radica en que, a pesar del contexto de los estudiantes y de sus familias, los profesores pueden enseñar y los estudiantes pueden aprender. Es decir, que la escuela no minimiza su responsabilidad por el hecho de trabajar en contextos de alta complejidad sociocultural (Weinstein y Muñoz, 2019). De esta manera, sus prácticas están puestas en el contex to y 
no en el déficit. Esto es relevante para entender que la mejora escolar no es solo focalizarse en el rendimiento escolar, aunque también lo es, sino entender los aprendizajes como parte de un constructo mayor que implica desarrollo profesional docente, cultura inclusiva, participación y vínculos afectivos entre profesores y estudiantes.

En síntesis, una escuela que persigue la inclusión debe tener una cultura que la desarrolle. A la vez, estas culturas deben contener una serie de prácticas que las promueva. Y, en parte, estas prácticas son responsabilidad de los equipos directivos. De esa manera, y en el caso de esta investigación, una escuela con alta cultura inclusiva tiene equipos directivos que desarrollan valores inclusivos, potencian procesos de enseñanza y aprendizaje para todos y se preocupan del desarrollo docente mediante una visión compartida. En contraste, la escuela con baja cultura inclusiva, aún no puede desarrollar estas prácticas, pues sus actuales preocupaciones están en la mejora de las relaciones humanas, aspecto que también es parte de una escuela inclusiva, pero que, si lo contrastamos con la escuela 1 , responde más a una fase inicial.

Las limitaciones de este estudio están en no contemplar la voz de los municipios, quienes mediante la administración de las escuelas chilenas se vuelven actores relevantes en el ámbito del liderazgo escolar. A futuro se sugiere realizar estudios que permitan evaluar el constructo de "liderazgo inclusivo" en escuelas chilenas y latinoamericanas. De esta manera, se abre un campo de estudio para comprender otras formas de liderazgo en contextos escolares que tienen a la inclusión como objetivo troncal de sus políticas públicas.

\section{Agradecimientos}

Este estudio se deriva de una tesis doctoral financiada por Conicyt mediante la beca doctoral $\mathrm{N}^{\circ} 21170256$.

\section{Referencias}

Agencia de Calidad de la Educación. (2017). Panorama de la gestión escolar ¿Cómo avanzamos en calidad en las escuelas que más apoyo requieren? Primer informe 2014-2015. Agencia de Calidad de la Educación.

Ashikali, T. y Groeneveld, S. (2015). Diversity management in public organizations and its effect on employees' affective commitment: The role of transformational leadership and the inclusiveness of the organizational culture. Review of Public Personnel Administration, 35(2), 146-168. https://doi.org/10.1177\%2F0734371X13511088

Bellei, C., Valenzuela, J. P., Vanni, X. y Contreras, D. (2014). Lo aprendí en la escuela: ¿Cómo se logran procesos de mejoramiento escolar? LOM.

Beneke, M. y Cheatham, G. (2016). Inclusive, democratic family-professional partnerships: (Re)conceptualizing culture and language in teacher preparation. Topics in Early Childhood Special Education, 25(4), 234-244. https://doi.org/10.1177\%2Fo271121415581611

Boekhorst, J. (2015). The role of authentic leadership in fostering workplace inclusion: A social information processing perspective. Human Resource Management, 54(2), 241-264. https://doi.org/10.1002/hrm.21669

Booth, T. y Ainscow, M. (2015). Guía para la educación inclusiva. Desarrollando el aprendizaje y la participación en los centros escolares. OEI. 
Carroll, B., Levy, L. y Richmond, D. (2008). Leadership as practice: Challenging the competency paradigm. Leadership, 4(4), 363-379. https://doi.org/10.1177\%2F 1742715008095186

Celik, S., Ashikali, T. y Groeneveld, S. (2013). Diversity interventions and employee commitment in the public sector: The role of an inclusive organizational culture. Gedrag en Organisatie, 26(3), 329-352. https://doi.org/10.1177\%2F0734371X13511088

Díaz de la Rada, A. (2013). Etnografía de la escuela más allá de la etnografía y de la escuela: Tensiones disciplinares y aplicabilidad de los saberes etnográficos. Educación y Futuro, 29, 13-39.

Dorczak, R. (2013). Inclusion through the lens of school culture. En G. Ruairc, E. Ottesen y R. Precey (Eds), Leadership for inclusive education (pp. 47-59). Studies in Inclusive Education. https://doi.org/10.1007/978-94-6209-134-4_5

Elmore, R. (2010). Mejorando la escuela desde la sala de clases. Fundación Chile.

Flick, U. (2012). Introducción a la investigación cualitativa. Paideia.

Gómez-Hurtado, I. (2014). El equipo directivo como promotor de buenas prácticas para la justicia social: Hacia un liderazgo inclusivo. Revista Internacional de Educación para la Justicia Social, 3(2), 141-159.

Gómez-Hurtado, I. (2013). Dirección y gestión de la diversidad en la escuela: hacia un liderazgo inclusivo. Revista Fuentes, 14, 61-84.

Leithwood, K., Harris, A. y Hopkins, D. (2008). Seven strong claims about successful school leadership. School Leadership \& Management, 28(1), 27-42. https://doi.org/10.1080/13632430701800060

León, M. J., Crisol, E. y Moreno, R. (2018). Las tareas del líder inclusivo en centros educativos de zonas favorecidas y favorecidas. REICE. Revista Iberoamericana sobre Calidad, Eficacia y Cambio en Educación, 16(2), 21-40. https://doi.org/10.15366/reice2018.16.2.002

León, M.J. (2012). El liderazgo para y en la escuela inclusiva. Educatio Siglo XXI, 30(1), 133-160.

López, V. y Valdés, R. (2018). Construcción y validación de instrumentos para evaluar prácticas de convivencia escolar en profesionales y padres. Revista Electrónica Actualidades Investigativas en Educación, 18(23), 1-29. https://doi.org/10.15517/aie.v18i3.34328

Ministerio de Educación. (2015). Marco para la buena dirección y el liderazgo escolar. Ministerio de Educación.

Moliner, O., Sales, A. y Escobedo, P. (2016). Posibilidades y limitaciones de generar cultura de centro desde las prácticas compartidas de aula: El caso de la educación intercultural inclusiva. Estudios sobre Educación, 30, 51-70. https://doi.org/10.15581/004.30.51-70

Ryan, J. (2017). Un liderazgo inclusivo para las escuelas. En J, Weinstein (Ed.), Liderazgo educativo en la escuela: Nueve miradas (pp. 177-204). Ediciones Universidad Diego Portales.

Spillane, J. y Ortiz, M. (2017). Perspectiva distribuida del liderazgo y la gestión escolar: implicancias cruciales. En J. Weinstein (Ed.), Liderazgo educativo en la escuela: nueve miradas (pp. 155-174). Ediciones Universidad Diego Portales.

Thorpe, A. y Bennett-Powell, G. (2014). The perceptions of secondary school middle leaders regarding their needs following a middle leadership development programme. Management in Education, 28(2), 52-57. https://doi.org/10.1177\%2F0892020614529808

Uribe, M. y Celis, M. (2012). Desarrollo de capacidades para un liderazgo escolar efectivo; del levantamiento de competencias al reconocimiento de buenas prácticas. En J. Weinstein y G. 
Muñoz (Eds). ¿Qué sabemos sobre los directores de escuela en Chile? (pp. 111-134). Pontificia Universidad Católica de Chile.

Valdés, R. (2018). Liderazgo inclusivo: La importancia de los equipos directivos en el desarrollo de una cultura de la inclusión. Revista de Investigación Educativa de la REDIECH, 9(16), 51-66. https://doi.org/10.33010/ie_rie_rediech.v9i16.73

Valdés, R. y Gómez-Hurtado, I. (2019). Competencias y prácticas de liderazgo escolar para la inclusión y la justicia social. Perspectiva Educacional, 58(2), 47-68. https://doi.org/10.4151/07189729-vol.58-iss.2-art.915

Valdés, R., López, V. y Chaparro, V. (2018). Convivencia escolar: Adaptación y validación de un instrumento mexicano en Chile. Revista Electrónica de Investigación Educativa, 20(3), 80-91. https://doi.org/10.24320/redie.2018.20.3.1720.

Vásquez, F. (1994). Análisis de contenido categorial: el análisis temático. Universitat Autònoma de Barcelona.

Waldron, N. y Mcleskkey, J. (2010). Establishing a collaborative school culture through comprehensive school reform. Journal of Educational and Psychological Consultation, 20(1), 5874. https://doi.org/10.1080/10474410903535364

Weinstein, J. y Muñoz, G. (2012). ¿Qué sabemos sobre los directores de escuela en Chile? Fundación Chile.

Weinstein, J. y Muñoz, G. (2019). Liderazgo en escuelas de alta complejidad sociocultural. Diez miradas. Ediciones Universidad Diego Portales.

Wenger, E. (2001). Comunidades de práctica. Aprendizaje, significado e identidad. Paidós.

\section{Breve CV del autor}

\section{René Valdés}

Profesor de Educación especial y Magíster en Educación especial y Psicopedagogía por la Universidad Católica del Maule y Doctor en Psicología por la Pontificia Universidad Católica de Valparaíso. Docente de la Escuela de Pedagogía de la Pontificia Universidad Católica de Valparaíso y de la Facultad de Educación y Ciencias Sociales de la Universidad Andrés Bello, Chile. Sus líneas de investigación son liderazgo escolar, inclusión e interculturalidad. Actualmente es investigador responsable del Fondecyt Postdoctorado $\mathrm{N}^{\circ}$ 3200192. ORCID ID: https://orcid.org/OOOO-0003-4242-9748. Email: rene.valdes@pucv.cl 